\title{
Solitons, chaos, and energy transfer in the Zakharov equations
}

\author{
F. B. Rizzato, G. I. de Oliveira, and R. Erichsen \\ Instituto de Física-Universidade Federal do Rio Grande do Sul, Caixa Postal 15051, 91501-970 Porto Alegre, Rio Grande do Sul, Brazil
}

(Received 10 July 1997)

\begin{abstract}
In the present paper we investigate the process of energy transfer in the Zakharov equations. Energy is initially injected into modes with small wave vectors. When the modulational instability threshold is exceeded, some additional modes with small wave vectors are excited and solitons are formed if one lies in a quasiintegrable regime and if the number of excited modes is large enough. These solitons are formed as a direct result of the modulational instability and in fact saturate the instability. However, use of a low-dimensional formalism based on collective variables shows that if the largest length scale of the linearly excited modes is much longer than the most unstable, these solitons may be greatly influenced as they interact with ion-acoustic waves. In those cases, full simulation of the space-time problem indicates that energy is progressively transferred to modes with very small length scales. Since we work with one spatial dimension, collapse is absent and energy transfer is due to the stochastic dynamics. [S1063-651X(98)02303-4]
\end{abstract}

PACS number(s): 05.45.+b, 52.35.Ra

\section{INTRODUCTION}

Langmuir turbulence has been one of the most extensively studied problems in modern nonlinear plasma physics. In recent years a great deal of effort has been directed to its analysis as well as to the analysis of related subjects of soliton dynamics, collapse, nucleation of cavitons, electromagnetic emission, and others [1]. More recently, attempts have been made to understand the turbulence in terms of concepts of nonlinear dynamics and chaos [2-6].

The conservative version of Langmuir turbulence is described by the Zakharov equations that couple the slowly varying amplitude of a high-frequency electric field, the Langmuir field, to slow density fluctuations, the ion-acoustic field. Decay processes deposit energy into fluctuations with long wavelengths and if the energy thus accumulated exceeds the threshold for modulational instability, transfer to modes with small wavelengths may be possible; we call this process energy transfer or redistribution of energy. However, even if the threshold is exceeded and the system becomes modulationally unstable, transfer may not occur. In integrable approximations of the Zakharov equations such as the nonlinear Schrödinger (NLS) equation, modulational instability may be present, but energy transfer is not; in this case the modulational instability is saturated when the linearly excited modes reorganize themselves into solitons and into some additional small-amplitude radiation. Alternatively, even if transfer is possible, there are some situations where the respective time scale is so large that is of little practical significance. In general, it is believed that some sort of chaotic process should be present to drive energy transfer from large to small spatial scales [7-10]. Chaotic activity would gradually destroy Kolmogorov-Arnold-Moser (KAM) surfaces, therefore enabling diffusion over large portions of the relevant phase space. Here we show that transfer may indeed occur in the context of the Zakharov equations and that it is faster when chaos is well developed in a subsystem that is constructed as a low-dimensional truncation of the full system. The identification of the appropriate lowdimensional subsystem depends on the range of parameters under analysis. Near the initial pitchfork bifurcation triggering the modulational instability [6], few modes are active and the subsystem is formed by a small number of plane waves undergoing nonlinear interaction; some of these waves are Langmuir fluctuations and some are ion-acoustic fluctuations. As one moves away from the bifurcation point, however, more and more modes become unstable and have an active participation in the dynamics. Then we find that a more suitable low-dimensional model should be constructed based on the nonlinear interaction of Langmuir solitons and ion-acoustic radiation.

The low-dimensional models inform that while within that region relatively near the initial pitchfork bifurcation mentioned above, the Langmuir modes are not much affected by the presence of the ion-acoustic waves. This is equivalent to saying that under such circumstances ion-acoustic fluctuations are unlikely to cause any chaos. This implies the existence of KAM surfaces in the low-dimensional model and the probable absence of energy transfer in the full multidimensional system. Moving farther away from the bifurcation, the first step is to commute from the plane-wave model to the low-dimensional model describing the interaction of solitons with ion-acoustic radiation. In this region, analysis of the respective phase space reveals that chaos becomes possible if, for a given amplitude of the initial pump wave, the largest length scale of the ion-acoustic field is larger than the soliton characteristic sizes. In this case, the soliton phase space is gradually more affected by ion-acoustic radiation until a mostly chaotic state sets in. The idea then is that if solitons of the low-dimensional model are essentially unaffected by the presence of ion-acoustic radiation, one does not have reasons to suspect that integrability will be broken in the full system since it is the action of nonadiabatic ionacoustic radiation that destroys this integrability. On the other hand, if solitons are affected, integrable features may not be present. In fact, precisely because they are strongly affected by ion-acoustic radiation, solitons may not survive mutual collisions and may emit continued radiation, which could cause energy flow from soliton length scales into smaller length scales. Full simulations confirm the above pic- 
ture. Therefore, based on the low-dimensional model and the results of full simulations, it is suggested that, provided the basic wave vector is small enough, chaos in the lowdimensional truncation and the associated energy transfer are present, even for small pump amplitudes.

As energy moves into modes with small wavelengths, dissipation becomes progressively more important. However, as we are interested only in nonlinear transfer processes, we discard dissipation in a first approximation. In any case, our result are of physical significance to related problems such as the Alfvenic turbulence. For this kind of turbulence where strong guiding magnetic fields are present, the system is described by similar equations; dissipation is weak [11] and the motion is mostly unidimensional [5] since the growth rate for fluctuations perpendicular to the ambient magnetic field is small. The model finds another application in pulsar radiation. In fact, the observed periodic radiation pulses emitted by these objects are believed to be formed when the amplitude of homogeneous trains of electromagnetic radiation crosses the instability threshold and causes the train to become modulationally unstable: In this kind of environment the dynamics is likely to be described by a nonlinear Schrödinger equation, which is a particular case of the equations analyzed here [12]. It is likely that in the presence of additional modes, energy transfer could take place here as well, which agrees with the fact that pulsar radiation actually contains an irregular component. Note that a different feature to be presented here is that since we work with a onedimensional system, energy transfer is not a result of soliton collapse. It is purely generated by the nonintegrable and chaotic aspects of the problem.

This paper is organized as follows. In Sec. II we introduce the basic equations and discuss the numerical techniques. In Sec. III we perform the appropriate low-dimensional estimates along with the full simulations. In Sec. IV we investigate energy transfer. In Sec. V we draw our final conclusions.

\section{BASIC EQUATIONS AND NUMERICAL TECHNIQUES}

The one-dimensional Zakharov equations governing the Langmuir turbulence can be written in the adimensional form [6]

$$
\begin{gathered}
i \partial_{t} E+\partial_{x}^{2} E=n E, \\
\partial_{t}^{2} n-\partial_{x}^{2} n=\partial_{x}^{2}|E|^{2},
\end{gathered}
$$

with $\partial_{t} \equiv \partial / \partial t$ and $\partial_{x} \equiv \partial / \partial x$. $E(x, t)$ is the slowly varying amplitude of the high-frequency Langmuir field and $n(x, t)$ are slow density fluctuations associated with the ion-acoustic field. The NLS equation

$$
i \partial_{t} E+\partial_{x}^{2} E+|E|^{2} E=0
$$

is obtained from the set of equations (1) and (2) if one is allowed to approximate Eq. (2) in order to replace $n$ with $-|E|^{2}+$ const. This approximation is called subsonic because it requires very slow time scales such that $\partial_{t}^{2} n(x, t)$ $\ll \partial_{x}^{2} n(x, t)$.
Our numerical approach is based on a pseudospectral method. We assume spatial periodicity with basic length $L$ and expand $E(x, t)$ and $n(x, t)$ into Fourier series as

$$
\begin{gathered}
E(x, t)=\sum_{m=-N / 2}^{+N / 2} E_{m}(t) e^{i m k x}=\sum_{m=-N / 2}^{+N / 2} \sqrt{\rho_{m}(t)} e^{i \phi_{m}(t)} e^{i m k x}, \\
n(x, t)=\sum_{m=-N / 2}^{+N / 2} n_{m}(t) e^{i m k x}
\end{gathered}
$$

The basic wave vector is defined in terms of the system length $L$ as $k=2 \pi / L$ and the amplitudes $\rho_{m}(t)$ along with phases $\phi_{m}(t)$ are conveniently introduced. The integer $N$ represents the variable number of modes used in the simulations. To represent a continuous system one should take the limit $N \rightarrow \infty$. In practice we set $N=256,512,1024$, and 2048 (this last value is used only in extreme cases) and remove half of the modes to cure aliasing problems associated with the fast Fourier transform routines. Accuracy is checked by varying the tolerance factor of the numerical integrator and by monitoring the conserved energy [1]. We find that relative fluctuations in energy are about one part in $10^{6}-10^{8}$ and that variations of the tolerance factor do not produce alterations in the outcome of runs.

\section{LOW-DIMENSIONAL MODELS VERSUS FULL SIMULATIONS}

Our system is multidimensional, but we would like to see whether a small subgroup of modes is more active than the remaining modes. If this is the case one could try to describe the basic features of the full dynamics by a low-dimensional approximation. As it turns out, such an approximation appears to be possible. However, we shall see that the specific details are highly dependent on the parameter range considered.

To see how to obtain the low-dimensional model, we proceed as follows. First we recall that as initial conditions we are interested in fluctuations with very long spatial scales. To represent this sort of configuration, in the simulations the initial long scales are simply implemented by a $k=0$ dipolar mode $E(x, t=0)=\sqrt{\rho_{o}(t=0)} \equiv \sqrt{\rho_{*}}$ and $n(x, t=0)=0$. The stability of the dipolar state against modulational instabilities can be examined if the state is perturbed with a small disturbance $\rho_{ \pm 1} \ll \rho_{*}$. A simple expression for the corresponding growth rate can be easily obtained in the subsonic regime where the characteristic frequency of the ion-acoustic waves, $\omega_{i a}=k$, is much larger than the one corresponding to the modulational instability [this is equivalent to taking $\partial_{t}^{2} n(x, t) \ll \partial_{x}^{2} n(x, t)$ in Eq. (2)]. We give some special relevance to the subsonic regime because it is the first regime appearing immediately after the pitchfork bifurcation destabilizing the system [6]. One finds for the subsonic growth rate $\Gamma$,

$$
\Gamma \sim k \sqrt{2 \rho_{*}-k^{2}},
$$

from which one sees that instability sets in only if $k<k_{u}$ $\equiv \sqrt{2 \rho_{*}}$, a relation defining the bifurcation curve $k=k_{u}$; it is along this curve that the pitchfork bifurcation mentioned ear- 
lier takes place [6]. We shall not need any further details on the bifurcation itself, so let us proceed to the study of the unstable states.

\section{A. The plane-wave region}

Below and sufficiently near the bifurcation curve such that $\sqrt{2 \rho_{*}-k^{2}}=\Gamma / k \ll 1$, the dynamics is really subsonic in the sense that the growth rate is much smaller than $k$. Below $k_{u} / 2$ the regime may no longer be subsonic if $\rho_{*}$ is relatively large, but informal use of Eq. (5) suggests that in that region not only is the mode with wave vector $k$ unstable, but also the mode with a higher harmonic wave vector $2 k$. Further reduction of $k$ gradually destabilizes more and more higher harmonics $[13,14]$. Therefore, a description in terms of a few active modes should be relatively accurate only if $k>k_{u} / 2$. In that region, even if not subsonic, the dynamics is still expected to be well described only in terms of the three linearly unstable modes for the electric field,

$$
E_{\text {trunc }}(x, t)=\sum_{m=-1,0,+1} \sqrt{\rho_{m}(t)} e^{i \phi_{m}(t)} e^{i m k x},
$$

and corresponding ion-acoustic fluctuations obtainable from

$$
\partial_{t}^{2} n-\partial_{x}^{2} n=\partial_{x}^{2}\left|E_{\text {trunc }}(x, t)\right|^{2} .
$$

We call the parametric region $k>k_{u} / 2$ the plane-wave region since it involves a small number of active Fourier modes; in other words, the dynamics involves a small number of interacting plane waves. Note that although we are truncating the modal expansion, no assumption on the slowness of dynamics is made. Of course if one assumes $\partial_{t}^{2} n \ll \partial_{x}^{2} n$, then one can only represent a subsonic dynamics. However, we intend to go further and examine how the system behaves when the subsonic validity conditions becomes progressively weaker. Indeed, we shall see that even for parameters located in the low-dimensional plane-wave region, the corresponding dynamics may not be trivially subsonic.

Let us then proceed to the study of the low-dimensional system, restricting our attention to even perturbations characterized by $\rho_{m}=\rho_{-m}, \phi_{m}=\phi_{-m}$, and $n_{m}=n_{-m}$. Our basic approximation at this point is that we truncate the modal expansion for the electric field up to terms with $|m|=1$ since modes with $|m|>1$ are stable; this is the key procedure to obtain a low-dimensional description. Ion-acoustic modes with $|m|=2$ are kept as they are, already excited in the presence of the $|m|=1$ electric fields. As a result we obtain a nonlinearly coupled system for the dynamical variables $\rho_{0}, \rho_{1}=\rho_{ \pm 1}, n_{1}=n_{ \pm 1}$, and $n_{2}=n_{ \pm 2}$. Some algebraic work shows that the nonlinear system so obtained can be derived from the truncated nonlinear Hamiltonian

$$
\begin{aligned}
H_{t r}= & -2 n_{1} \sqrt{2 \rho_{0} \rho_{1}} \cos \left(\phi_{0}-\phi_{1}\right)+\frac{1}{2} n_{2} \rho_{1}+k^{2} \rho_{1} \\
& -\frac{1}{2}\left(k^{2} p_{1}^{2}+n_{1}^{2}\right)-\frac{1}{2}\left(4 k^{2} p_{2}^{2}+n_{2}^{2}\right),
\end{aligned}
$$

where scales are changed according to $\rho_{0} / 2 \rightarrow \rho_{0}$ and $2 \phi_{0} \rightarrow \phi_{0}$ (a similar Hamiltonian describing decay instabilities has been derived recently [15]). In the Hamiltonian (8) the variables $\rho$ are to be considered momenta conjugate to coordinates $\phi$ and the variables $p$ are to be considered momenta conjugate to the density fluctuations $n$. The structure of the Hamiltonian suggests a canonical transformation that introduces $\psi \equiv \phi_{0}-\phi_{1}$ as a new angular coordinate; the new amplitudes are written correspondingly in the form $\rho_{0} \rightarrow \rho_{0}$ and $\rho_{1} \rightarrow \rho_{1}-\rho_{0}$. Due to the absence of $\phi_{1}$ from the transformed Hamiltonian, $\rho_{1}$ is now a constant that we identify as the maximum value of $\rho_{0}$ for a given energy, i.e., $\rho_{*}$. To summarize, the Hamiltonian (8) is a low mode truncation of the full dynamics. If one lies within the plane-wave lowdimensional region of the parameter space, it can be expected to yield an accurate representation of the actual dynamics. So let us take a brief look at its integrability characteristics.

If all the relevant amplitudes are small, one could initially suspect that terms coupling $n$ 's and $\rho$ 's are smaller than the others. Then one could define an action-angle representation in the form $I, \theta$ for the ion-acoustic waves and perform standard calculations. However, this procedure is not convenient here as it does not indicate directly any resonance. In fact, under these conditions it can be easily seen that $\dot{\psi} \sim k^{2}$ and $\dot{\theta} \sim k$; due to the disparity of frequencies for $k \ll 1$, no primary resonant coupling is possible. To detect the presence of resonances and thus chaos, one should realize from the Hamiltonian that small $\rho$ 's evolve very slowly in time. In fact, their time scales are much longer than those associated with the ion-acoustic modes. This type of behavior is the adiabatic regime so well known in plasma physics. To handle the adiabatic Hamiltonian one simply redefines the origin for the ion-acoustic oscillators so as to place both at the slowly varying minimum of the effective potential acting on each. The new origin for $n_{1}$ (we call it $n_{1, a d}$ ), for instance, is obtained from relation $\dot{p}_{1}=2 \sqrt{2 \rho_{0}\left(\rho_{*}-\rho_{0}\right)} \cos \psi$ $+n_{1, a d}\left(\rho_{0}, \psi\right) \approx 0$ and a similar relation holds for $n_{2}$. One then introduces a canonical transformation with generating function of the form $S=\left[n_{1}-n_{1, a d}\left(\rho_{0}^{\prime}, \psi\right)\right] p_{1}^{\prime}+\left[n_{2}\right.$ $\left.-n_{2, a d}\left(\rho_{0}^{\prime}, \psi\right)\right] p_{2}^{\prime}+\psi \rho_{0}^{\prime}$ (primes on new coordinates) to shift the origin as discussed. The rules of canonical transformation and an appropriate expansion of $\rho_{0}^{\prime}$ in the argument of $n_{1, a d}$ and $n_{2, a d}$ leads to the form (primes dropped)

$$
\begin{aligned}
H_{t r}= & -2 n_{1, a d}\left(\rho_{0}, \psi\right) \sqrt{2 \rho_{0}\left(\rho_{*}-\rho_{0}\right)} \cos \psi+\left[\frac{1}{2} n_{2, a d}\left(\rho_{0}, \psi\right)\right. \\
& \left.+k^{2}\right]\left(\rho_{*}-\rho_{0}\right)+k J_{1}+2 k J_{2}+H_{c}\left(J_{i}, \rho_{0}, \psi, \theta_{i}\right) \\
\equiv & H_{a d}(\rho, \psi)+k J_{1}+2 k J_{2}+H_{c}
\end{aligned}
$$

where $J_{i}$ and $\theta_{i}(i=1,2)$ are action-angle coordinates associated with the new ion-acoustic variables and the small term $H_{c}$, whose explicit form we do not work out here, contains all the nonlinear couplings involving the new $\rho$ 's and $n$ 's. $H_{a d}$, on the other hand, is the integrable Hamiltonian controlling the $\left(\rho_{0}, \psi\right)$ dynamics in the strictly adiabatic regime where the large differences of time scales cause the averaged $H_{c}$ to vanish. The Hamiltonian $H_{t r}$ is three degrees of freedom. There are relatively precise techniques to extract information from such a kind of system, but here we follow a simpler way and analyze the two-degrees-of-freedom version $H_{t w o}=H_{a d}+k J_{1}+H_{c}$ that is obtained when one assumes that the second ion-acoustic wave is weakly excited $J_{2} \approx 0$. 

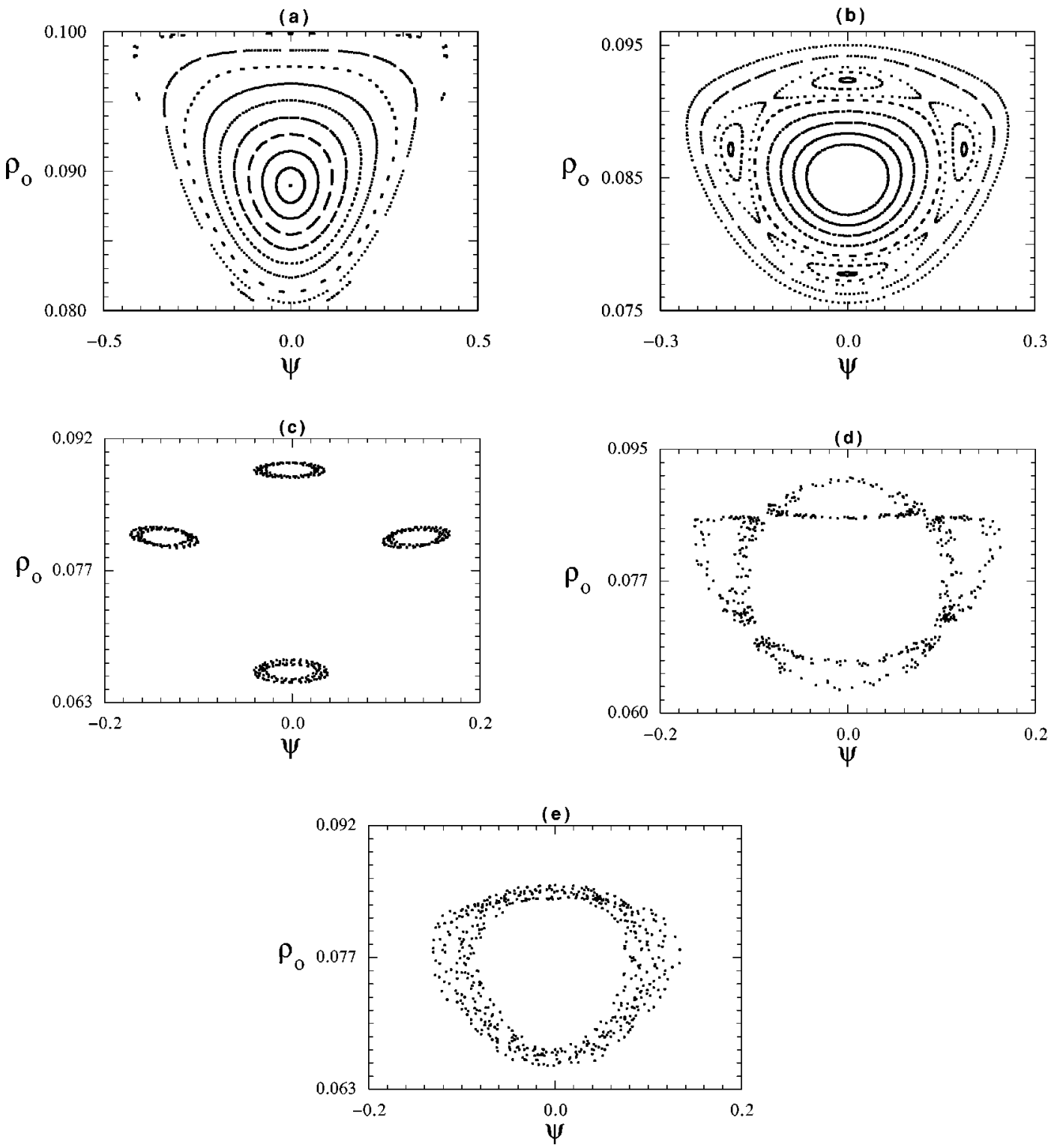

FIG. 1. Poincaré plots $\left(\rho_{0}, \psi\right)$ of the plane-wave model for (a) $k=k_{u} / 1.001$ and (b) $k=k_{u} / 1.12$. In (c)-(e) we display Poincaré plots obtained from the full simulation for $k=k_{u} / 1.11$. Several initial conditions are shown, indicating the correctness of the low-dimensional plane-wave model. Angles are given in radians and $\rho_{*}=0.1$.

This assumption yields good agreement with the simulations. If Fig. 1 we compare Poincare plots of $H_{t w o}$ and of the full system, recording the values of $\rho_{0}$ and $\psi$ each time $p_{1}=0$, $\dot{p}_{1}>0$; we choose $\rho_{*}=0.1$; for much smaller values of the pump amplitude we could not find resonances in this planewave region. Close to the bifurcation curve $k_{u}$, as in Fig. 1(a) where we take $k=k_{u} / 1.001$, the low-dimensional dynamics is well approximated by smooth curves. It should not be surprising that those smooth curves are in fact the curves of constant $H_{a d}$. The rotational frequency around the central elliptic point is denoted by $\Omega_{0}$; it scales like $\Omega_{0} \sim \sqrt{\rho_{*}}$. As we decrease $k$, smoothness of $H_{t w o}$ breaks down; this is seen in the form of resonant islands in Fig. 1(b), where the lowdimensional dynamics is mapped with $k=k_{u} / 1.12$. For comparison, in Fig. 1(c) we display the corresponding plot obtained from full simulation with $N=256$, considering $k$ $=k_{u} / 1.11$. In both cases (b) and (c), fourth-order resonances are seen; the Hamiltonian $H_{t w o}$ therefore explains self- consistently some of the resonance features detected in recent simulations [4,6]. In addition, Figs. 1(d) and 1(e) show that for initial conditions other than the one corresponding to the resonance chain, a separatrix and quasiperiodic orbits are generated, which agrees with the general topology of Fig. 1(b). Note that there is some finite thickness associated with the plots produced with full simulations. This is a signature of the multidimensional aspect of the problem; if the system were genuinely two degrees of freedom, as the lowdimensional model is, the curves would be of zero thickness as in Figs. 1(a) and 1(b). All in all, the final conclusion is that the model explains the dynamics well.

The regularity of Fig. 1 suggests that within the planewave region $k>k_{u} / 2$, strong chaotic activity is absent. Most of the KAM surfaces, and especially the central fixed point, are not affected at all by the nonlinear interaction. In fact, strong chaos is absent not only for this particular value of $\rho_{*}$ but for any other; for large $\rho_{*}$, in particular, all resonances 
disappear. This suggests that KAM surfaces are globally preserved within the plane-wave region and that no massive redistribution of energy occurs if one takes basic wave vectors inside that region. This information, which will be confirmed later, is relevant since various simulations use as the basic wave vector the one providing maximum growth rate for the initial modulational instability, $k=k_{\max } \equiv k_{u} / \sqrt{2}$. This wave vector lies inside the low-dimensional plane-wave region and the corresponding simulations cannot reveal the full statistical and chaotic character of the Zakharov equations.

Although the plane-wave dynamics does not explicitly indicate chaotic activity, it suggests where to look for it. From Fig. 1 we see that as $k$ is lowered, integrable approximations tend to become poorer. As a matter of fact, the presence of resonant islands in the situation of Fig. 1(b) shows that some small chaos is likely to be present due to the formation of separatrices. So it may be possible that as the basic wave vector is decreased further, strong chaotic activity develops in the system. The problem is that to make any estimate when $k$ is too small, one could not comfortable rely on expansions containing only a small number of modes, as we did previously, because if $k$ is too small many modes become linearly unstable. Our attempt to produce low-dimensional estimates should be appropriately corrected as we do in the next subsection.

\section{B. The soliton region}

When $k \ll k_{u} / 2$, many Langmuir modes are destabilized simultaneously. Recalling that $k_{\max }=k_{u} / \sqrt{2}$ is the wave number of the most unstable mode, the initial modulational instability tends to produce $k_{\max } / k$ bumps along the spatial axis. Most of the bumps evolve into solitons, a feature that allows us to estimate this soliton number $N_{s}$ simply as $N_{s}$ $\sim k_{\max } / k \sim k_{u} / k$, which is also approximately the number of linearly unstable modes. Were the system completely integrable as in the case of its NLS approximation, the final asymptotic state would be just the collection of solitons formed with these many unstable modes. Our full Zakharov system is not integrable, but a reasonable hypothesis would be the following. The homogeneous dipolar state first decays into the collection of solitons as it would in the integrable approximation. However, in addition to the collection of solitons, a certain amount of free ion-acoustic radiation is now produced, where by free we mean that portion of the radiation not adiabatically enslaved to the Langmuir field. Eventually, the ion-acoustic radiation interacts with the solitons with the following possibilities. (i) The interaction is weak such as not to greatly influence the soliton dynamics. Then one may expect that the overall system is accurately described by the integrable NLS equation. (ii) The interaction is strong enough to greatly distort soliton dynamics. Then the solitons either can be directly destroyed by the ion-acoustic radiation or may not survive mutual collisions among themselves, again because the ion-acoustic radiation is strong enough to inhibit the appearance of all the nice integrability and invariance properties known to be satisfied in soliton-soliton collisions.

The idea of homogeneous states decaying into Langmuir solitons has been used in a paper by Shen and Nicholson
[17]. Here we add ion-acoustic free radiation to see how far the integrable picture and the concept of stable solitons are accurate. If solitons become unstable by virtue of the ionacoustic waves, it is likely that the energy initially placed in the dipolar state may find its way into fluctuations with smaller and smaller length scales. Otherwise, if the initial instability is effectively saturated by soliton formation, one could expect that the energy flux triggered by the initial modulational instability is arrested when solitonic length scales are attained. One could also argue that even when solitons have not been completely affected by the ionacoustic effects they might still deliver energy irreversibly to the remaining modes of the system, provided the soliton dynamics is sufficiently chaotic. According to this stochastic pump model [16], as soon as a strongly chaotic subset of degrees of freedom of a conservative dynamical system is coupled to an environment, the chaotic subset acts like a pump delivering a neat amount of energy to its vicinity. This neat energy transfer is diffusive and takes place at all because of the very random nature of the coupling. These are issues to be discussed later. Before that, let us proceed with the construction of our low-dimensional model where now solitons, instead of plane waves, interact with free ionacoustic radiation.

To construct the low-dimensional model, we make use of the techniques of averaged Lagrangians [18]. In other words, we start with the Lagrangian of the full system and replace the various fields with appropriate solitonic functions where parameters such as amplitude and phase are allowed to vary in time. Euler-Lagrange equations are then used to obtain the temporal dynamics of these slowly varying parameters. In addition to the soliton fields, we also include free ionacoustic radiation. As a final result we expect to obtain a low-dimensional set of equations describing the nonlinear interaction involving the soliton parameters and those characterizing the radiation.

The full Lagrangian of our system reads

$$
\begin{aligned}
L= & \int \mathcal{L} d x \equiv \int\left[\frac{i}{2}\left(E^{*} \partial_{t} E-E \partial_{t} E^{*}\right)-\left|\partial_{x} E\right|^{2}-|E|^{2} \partial_{x} \nu\right. \\
& \left.+\frac{1}{2}\left[\left(\partial_{t} \nu\right)^{2}-\left(\partial_{x} \nu\right)^{2}\right]\right] d x
\end{aligned}
$$

where the dynamical variable $\nu(x, t)$ is introduced in the form $n(x, t) \equiv \partial_{x} \nu(x, t)$. The Euler-Lagrange equation for $E(x, t)$, for instance, is written as

$$
\partial_{t} \frac{\partial \mathcal{L}}{\left(\partial_{t} E\right)}=\frac{\partial \mathcal{L}}{\partial E}-\partial_{x} \frac{\partial \mathcal{L}}{\partial\left(\partial_{x} E\right)},
$$

with similar expressions holding for the other variables. From expression (11), in particular, one obtains Eq. (1). As mentioned before, what has to be done now is to substitute into Eq. (10) a one-soliton solution plus ion-acoustic radiation to examine the temporal dynamics of the solitonic parameters. For the soliton we choose a solution of the form

$$
E_{s}(x, t)=\sqrt{2} \eta(t) \operatorname{sech}\left(\frac{x}{\alpha(t)}\right) e^{i \Phi(t)}
$$


If $\eta=1 / \alpha$ and $\Phi=\eta^{2} t / 2$ one has a stationary solitary solution of the Zakharov equations. Note that we consider solitons with zero velocity. Previous works [17] and the full simulations to be performed here indeed suggest negligible subsonic velocities with magnitudes on the order of 0.01 times the sound velocity. This leads us to conclude that as far the one-soliton dynamics is concerned, the dominant dynamical effect is the breathing feature related to the time dependence of $\eta$ and $\alpha$.

As for the ion-acoustic radiation, the choice follows the following reasoning. First we note from Eq. (2) that a convenient way to write the ion-acoustic field should be

$$
n(x, t)=-|E|^{2}+\mu(x, t) .
$$

A global constant factor guaranteeing that the spatial average of $n(x, t)$ vanishes has been absorbed into $E$ as a phase factor. In other words, what we do here is split the field into its adiabatic response to the soliton field, the first term on the right-hand side, and an additional term, $\mu(x, t)$, that takes into account the nonadiabatic features; actually, we have been referring to the nonadiabatic dynamics as the free ionacoustic field throughout the paper. In the purely adiabatic case, $\mu \rightarrow 0$. We then replace $E$ with $E_{s}$ in Eq. (13) and proceed to the second step, which is to determine a convenient form for $\mu(x, t)$. In order to accomplish this, we start by noticing that the ion-acoustic field in principle should be written as a summation over various Fourier modes like in the case of the Langmuir field:

$$
n(x, t)=-\left|E_{s}\right|^{2}+\mu(x, t)=\sum_{m}\left[-\left(\left|E_{s}\right|^{2}\right)_{m}+\mu_{m}(t)\right] e^{i m k x} .
$$

One then combines Eqs. (2) and (4) to obtain

$$
\ddot{n}_{m}+(m k)^{2}\left[n_{m}+\left(|E|^{2}\right)_{m}\right]=0 .
$$

The idea now is to produce an estimate for the nonadiabatic fluctuations of the ion-acoustic field as solitons are formed. Once we have the estimate, the dynamics evolving from this initial configuration can be described in terms of the average Lagrangian technique. In more concrete terms, to determine the initial conditions for the nonadiabatic ion-acoustic field, we assume that the Langmuir field initially evolves into an intermediary metastable state formed by the solitons, before any slow energy transfer has any chance to operate. The saturated value of $\left|E_{m}\right|^{2}$ in this state can be approximately evaluated as $\left|E_{m}\right|^{2} \sim \rho_{*} / 2 N_{s}$ if $m$ is such that $k<|m| k<k_{u}$ and $\left|E_{m}\right|^{2} \sim 0$ otherwise. To derive these last relations one simply makes use of the conserved integral

$$
\int_{-L / 2}^{L / 2}|E(x, t)|^{2} d x
$$

considers $E(t=0)=\sqrt{\rho_{*}}$, and supposes a relatively flat spectrum if $k<|m| k<k_{u}$ (see Ref. [17]); the flat spectrum, in particular, is appropriate to describe a field formed by a collection of $N_{s}$ solitons. However, what we really want is $\left(|E|^{2}\right)_{m}$ and not $\left|E_{m}\right|^{2}$. The connection is complicated, but if $m$ is small and the number of modes is relatively large one has, in terms of order of magnitude,
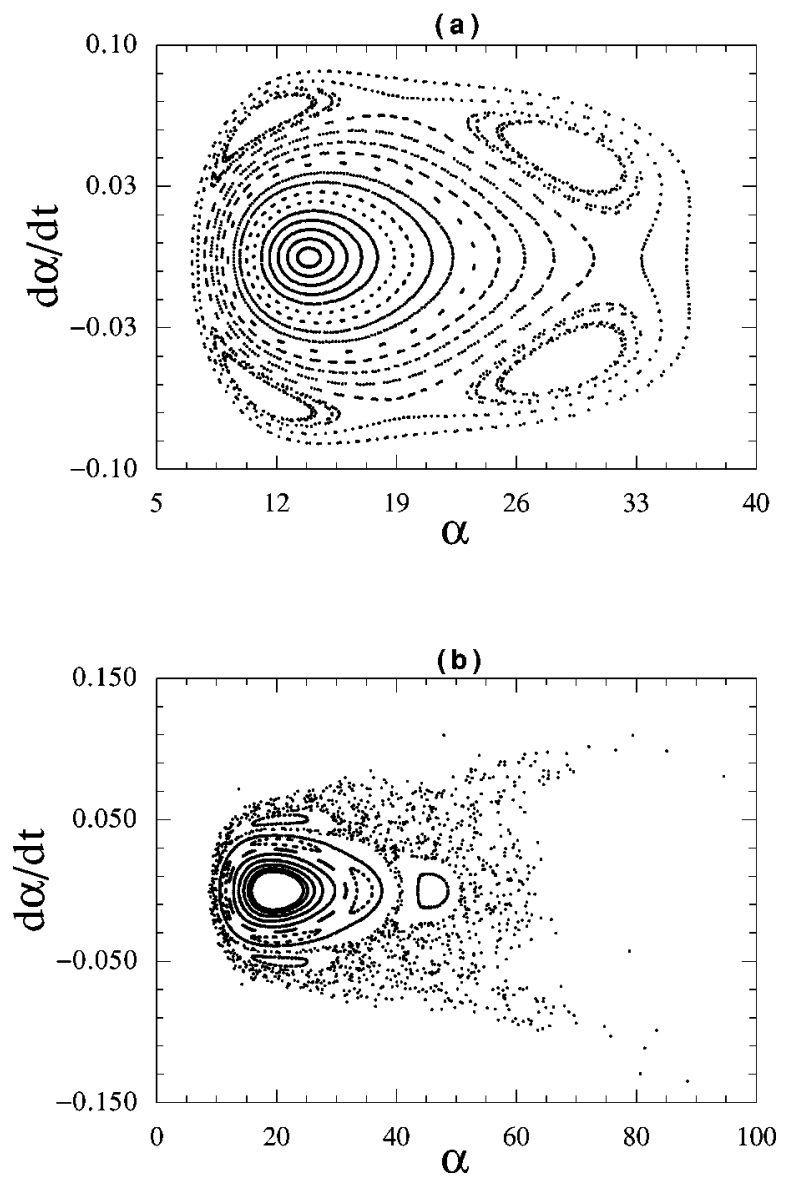

FIG. 2. Comparison of spectral distributions for $\mu$ and $|E|^{2}$ for (a) $k=k_{u} / 4$ and (b) $k=k_{u} / 20 . \rho_{*}=0.01$.

$$
\left(|E|^{2}\right)_{m}=\sum_{j} E_{j} E_{j-m}^{*} \sim \sum_{j}\left|E_{j}\right|^{2} \sim 2 N_{s}\left(\rho_{*} / 2 N_{s}\right)=\rho_{*} ;
$$

for larger values of $m,\left(|E|^{2}\right)_{m} \rightarrow 0$. Then we notice again that Eq. (15) is the equation of an oscillator with a moving origin given by $-\left(|E|^{2}\right)_{m}$. Therefore, if the fastest growth rate of $E$ towards the metastable state is much faster than the oscillator frequency $m k$, the amplitude of the oscillatory motion described by $\mu_{m}$ around the displaced origin is the nonadiabatic component of the ion-acoustic field and can be written as

$$
\mu_{m} \sim\left(|E|^{2}\right)_{m} \sim \rho_{*}
$$

if $m$ is small. In practice the adiabatic approximation breaks down when the slowest ion-acoustic frequencies $\omega_{i a} \sim|m| k$ ( $|m|$ small) become comparable to the fastest growth rate of the modulational instability $\Gamma_{\max } \sim \rho_{*}$; for $\rho_{*}=0.01$ this yields $k \sim 0.01 \sim k_{u} / 14$. Figure 2 shows the spectral distribution of the adiabatic and nonadiabatic densities $\mu_{m}$ and $\left(|E|^{2}\right)_{m}$ respectively, for $k=k_{u} / 4$ [Fig. 2(a)] and $k=k_{u} / 20$ [Fig. 2(b)]. The time elapsed in both cases is twice the growth period of the fundamental harmonic $1 / \Gamma(k)$, with $\Gamma(k)$ given in Eq. (5). It is seen that while in Fig. 2(a) the nonadiabatic density is very small, it really grows in magnitude as $k$ is lowered as depicted in Fig. 2(b); in terms of order of magnitude, the numbers agree with the estimates. 
The ion-acoustic fluctuations around the moving origin are the nonadiabatically enslaved fluctuations we want. Now that we have the initial condition for the nonadiabatic component of the ion-acoustic field, let us consider a number of options in regard to the interaction in our soliton model. One of them would be to consider a model in which the soliton interacts self-consistently with the entire spectrum of ion-acoustic modes, but in this case our model would lose its lowdimensional simplicity. Another option would be to consider the ion-acoustic field as externally given and fixed by the initial conditions and examine the soliton dynamics under this external drive, but in this case we would not have a self-consistent treatment. Yet another option would be to model the ion-acoustic field only with one Fourier component. This one component would be the basic one since it is the first for which the adiabatic approximation fails. In this case it would not be hard to construct a self-consistent model, although we would not be providing a very accurate description of the ion-acoustic spectral distribution and its total energetic content. At any rate, we prefer to follow this last alternative because when the system becomes chaotic, a strong energy exchange between Langmuir and ion-acoustic fields takes place, a feature in principle requiring selfconsistency. As it turns out, both the self-consistent model and the model with external driving furnish very similar results about the transition to chaos, which leads us to think that however crude, these models are robust.

We therefore write the complete ion-acoustic field in the form

$$
n=-\left|E_{s}\right|^{2}+A(t) \cos (k x),
$$

where $A(t)$, to be evaluated later, is the time-dependent amplitude of the nonadiabatic contribution.

On substituting relations (12) and (19) into the Lagrangian (10) one finally obtains

$$
\begin{aligned}
\mathcal{L} \approx & -2 W \dot{\Phi}+N_{s}\left[\frac{4 W^{2}}{3 \alpha}-\frac{2 W}{3 \alpha^{2}}+0.429 \frac{W^{2} \dot{\alpha}^{2}}{\alpha}-3.290 W \dot{\alpha} \alpha \dot{A}\right] \\
& +\frac{\pi \dot{A}^{2}}{k^{3}}-\frac{\pi A^{2}}{k}
\end{aligned}
$$

with $W=\eta^{2} \alpha$. The presence of the factor measuring the number of solitons $N_{s}$ is an attempt to incorporate the idea that the ion radiation is in fact interacting with this group of $N_{s}$ solitons. The various numerical factors appear in Eq. (20) as a result of the integrals involving trigonometric and hyperbolic functions.

The Euler-Lagrange equation with respect to the variable $\Phi$ indicates that $W$ is a constant of motion. As a matter of fact, this feature has been used already to simplify the form of the Lagrangian (20) by dropping terms proportional to $\dot{W}$ up to some positive power. Euler-Lagrange equations are then applied to variables $\alpha$ and $A$ to produce a two-degreesof-freedom conservative dynamical system. Interestingly, if we set $A \rightarrow 0$ we may still have oscillatory solutions without the necessity of introducing chirp factors [19]. This residual time dependence is a result of the ion-acoustic $\left(\partial_{t} \nu\right)^{2}$ term retained in the full Lagrangian. In other approximations where the ion-acoustic adiabatic dynamics is completely dis-
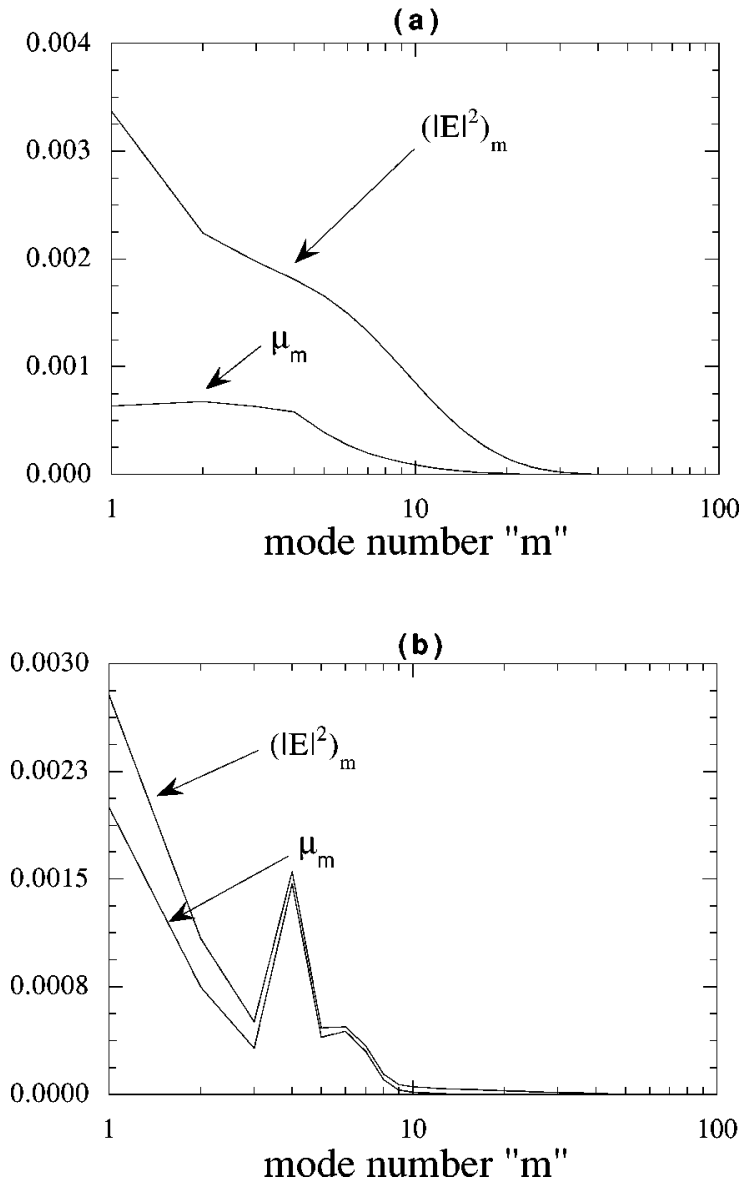

FIG. 3. Poincaré plots $(\dot{\alpha}, \alpha)$ of the soliton model for (a) $k$ $=k_{u} / 4$ and $(\mathrm{b}) k=k_{u} / 20 . \rho_{*}=0.01$.

regarded, i.e., $\partial_{t} \nu \rightarrow 0$, chirp factors are introduced, creating the appropriate time dependences.

We now examine what happens when we lower the wave vector $k$. Under this circumstances, and just to recall what we said earlier, it is expected that solitons are progressively more affected until they are completely destroyed as $k$ becomes smaller. In Fig. 3 we make Poincare plots on the $(\alpha, \dot{\alpha})$ plane for decreasing values of $k$; points are recorded each time $\dot{A}=0$ with $\ddot{A}>0$. The amplitude of one isolated soliton is calculated on basis of the conserved integral (16) when one again assumes that an initial dipolar state of amplitude $E_{0}=\sqrt{\rho_{*}}$ decays into $N_{s}$ identical solitons. As mentioned before, the effective initial amplitude $A(t=0)$ is evaluated as $A(t=0)=\rho_{*}$ and is associated with the outermost orbit of the figures, which is the last trapped and most vibratory one. This orbit is presumably the one into which the homogeneous state first decays. The amplitudes associated with the other orbits are obtained under the condition that the energy of all orbits be the same. The estimates are rough, but their qualitative and even quantitative results are in agreement with the full simulations. Let us then discuss the behavior depicted in Fig. 3, where we plot the phase spaces corresponding to $k=k_{u} / 4$ [Fig. 3(a)] and $k=k_{u} / 20$ [Fig. 3(b)]. For simplicity we use the nonadiabatic estimate $A \sim \rho_{*}$ even in the case $k=k_{u} / 4$, which is not quite true, but even then chaos is absent.

What is seen is that the dynamics undergoes a gradual 
transition to chaos as $k$ decreases. For relatively small values of $k$ like in Fig. 3(b), where we take $k=k_{u} / 20$, separatrix chaos is already substantial; the term "separatrix chaos" may no longer even be precise in this case since chaotic orbits are already spreading over the entire phase space. Here, although the central fixed point keeps its integrity, one may expect that the irregular orbits developing close to the separatrix act as the stochastic pump mentioned earlier [16]. What one would be likely to see in the full system under stochastic pump conditions is a gradual and slow transfer of energy from modes with relatively large length scales (the ones used in the formation of solitonic structures) to modes with smaller and smaller length scales (the scale where wave energy would be transferred to particles had dissipation been included in the model). This slow energy transfer is also called Arnold diffusion. Of course, as solitons deliver their energy they would tend to gradually disappear after some amount of time, a feature that also should be present in the simulations.

Energy transfer shall be analyzed later, but appropriate figures obtained with full simulations already give support to this idea of slowly modifying solitons. It is hard to measure numerically parameters of individual solitons in the numerical runs, when there is a large number of such entities along the spatial axis. Nevertheless, the simulations analyzed in Fig. 4 suggest that the present low-dimensional point of view is reasonable. In the figures we display three-dimensional plots [in Fig. 4(a)] and contour plots (in the other figures) for $|E(x, t)|^{2}$, considering a homogeneous initial condition with $\rho_{*}=0.01$ perturbed by small fields $\rho_{ \pm 1}=\rho_{*} \times 0.01$; in Figs. 4(a) and 4(b) we take $k=k_{u} / 4$ and in Fig. 4(c), $k=k_{u} / 20$. In all cases the simulation time corresponds to 20 times the growth periods of the basic mode $k$; the spatial dependence is represented on the simulation grid according to the scale shown in the figures and the coordinate $x=0$ corresponds to the grid position 512. $N=1024$ modes have been used in this particular set of simulations. While for $k=k_{u} / 4$ solitary structures are always seen moving along the spatial axis, for $k=k_{u} / 20$ solitons are strong and can be identified only during the initial period of time. The central stationary soliton, in particular, disappears right after a collision that sets it into a few oscillations. In general, as times progresses, solitons lose their strength and some time after 10-15 growth periods the space-time sharp and regular structures can hardly be seen. As solitons gradually vanish, irregular radiation increases, which agrees with the stochastic pump mentioned earlier. In Fig. 4(d) we show the contour plot corresponding to $k=k_{u} / 40$. It is seen that indeed the lifetime of solitons becomes shorter as $k$ is lowered further.

The central stationary soliton has zero velocity, but even moving solitons are slow: Their typical velocity is 0.01 . This reinforces the idea that velocity effects are negligible since during their lifetime solitons move only once or twice along the system length. On the other hand, after 50-100 free ionacoustic periods, the low-dimensional soliton model for $\rho_{*}$ $=0.01$ has revealed intense chaotic activity due to breathing. This amounts to a time interval ranging from 10 to 20 growth periods, the range within which solitons are actually seen to disappear in the simulations. Finally, in Fig. 4(e) we display the contour plot obtained from the simulation of the NLS equation with $k=k_{u} / 20$. The idea here is to use the NLS equation as an integrable reference to the more complicated case of the Zakharov equations. What we see is that, as opposed to the previous Zakharov cases, here NLS solitons are virtually indestructible, as they should be. Comparisons between Zakharov and NLS equations are useful and should be used further in the next section.

\section{ENERGY TRANSFER}

It is now of interest to measure energy transfer and the number of modes participating actively in the nonlinear interaction. Considering the features represented in Fig. 4 and all the previous discussions, one would expect that the number of active modes of Figs. 4(a) and 4(b) remains small while the number of active modes of Fig. 4(c), for instance, displays a substantial growth as time evolves. Speaking more precisely, due to the slight aperiodicity of Figs. 4(a) and 4(b), and according to our discussion on Arnold diffusion, a slow growth could be expected there, but nothing as sharp as what is expected to happen in Fig. 4(c).

We numerically measure the number of active modes with basis on the spectral average defined by [14]

$$
\left\langle N^{2}\right\rangle \equiv \frac{\sum_{m} m^{2} \rho_{m}}{\sum_{m} \rho_{m}} .
$$

$\sqrt{\left\langle N^{2}\right\rangle}$ yields an estimate of the active Langmuir modes and is evaluated for both the NLS $\left(\sqrt{\left\langle N^{2}\right\rangle}\right.$ NLS $)$ and Zakharov $\left(\sqrt{\left\langle N^{2}\right\rangle_{Z}}\right)$ equations. We know that in the NLS case, the number of modes does not grow beyond that predicted by linear theories $[13,14]$; the NLS solitons are precisely constructed with these linearly unstable modes. In the case of the Zakharov equations, on the other hand, we expect the behavior outlined in the preceding paragraph: If $k$ is relatively large, $\sqrt{\left\langle N^{2}\right\rangle_{Z}}$ and $\sqrt{\left\langle N^{2}\right\rangle_{N L S}}$ are expected to behave similarly, except perhaps for a slow upward drift of $\sqrt{\left\langle N^{2}\right\rangle_{Z}}$, a feature connected to the slow Arnold diffusion; if $k$ diminishes $\sqrt{\left\langle N^{2}\right\rangle}$ is expected to develop a substantial growth in time.

In Fig. 5(a) we examine the spectral average when $k$ $=k_{u} / 4$. After a fast initial growth commanded by the linear instability, the number of active modes of both NLS and Zakharov equations reaches saturation, except for the very slow, almost imperceptible, residual increase of $\sqrt{\left\langle N^{2}\right\rangle_{Z}}$. This agrees with the regularity displayed in Figs. 3(a), 4(a), and 4(b). If $k$ is lowered down to $k=k_{u} / 20$, the fast initial growth connected to the linear instability is again present in both the NLS and Zakharov cases. Now, however, after the initial linear growth, $\sqrt{\left\langle N^{2}\right\rangle_{Z}}$ continues to increase as time evolves: $\sqrt{\left\langle N^{2}\right\rangle_{N L S}}$ remains small and saturated as it should be. This continued growth of $\sqrt{\left\langle N^{2}\right\rangle_{Z}}$ is not as fast as the linear growth, but is surely much faster than the one corresponding to the previous case where we took $k=k_{u} / 4$. Note that in this case of $k=k_{u} / 20$, both Figs. 3(b) and 4(c) indicate that an appreciable degree of aperiodicity is already present. Figure 3(b), in particular, shows an already very extended separatrix chaos. In addition, the figures suggest that there is no sharp transition between the NLS regime and a genuine Zakharov regime. As it appears, a temporal growth of $\sqrt{\left\langle N^{2}\right\rangle_{Z}}$ is always present, satisfying the rule that the higher 
(a)

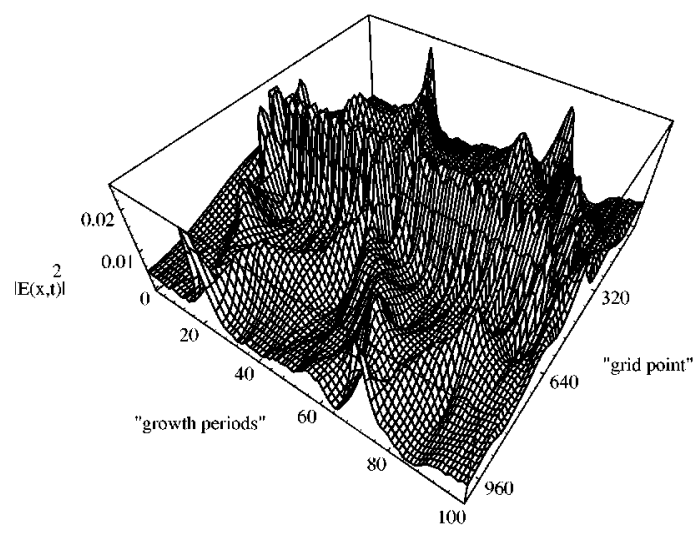

(b)

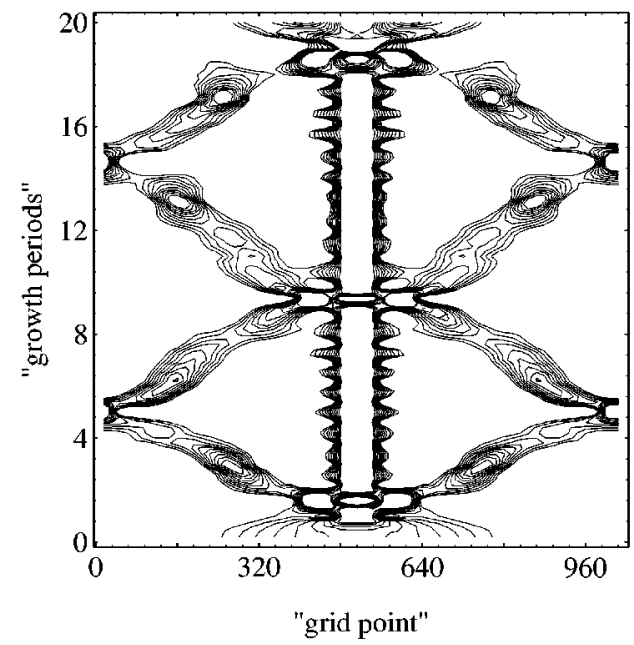

(c)

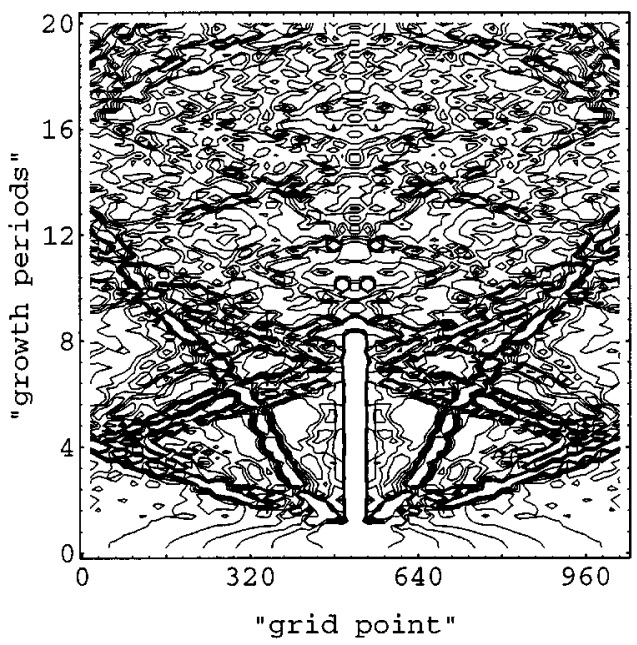

(d)

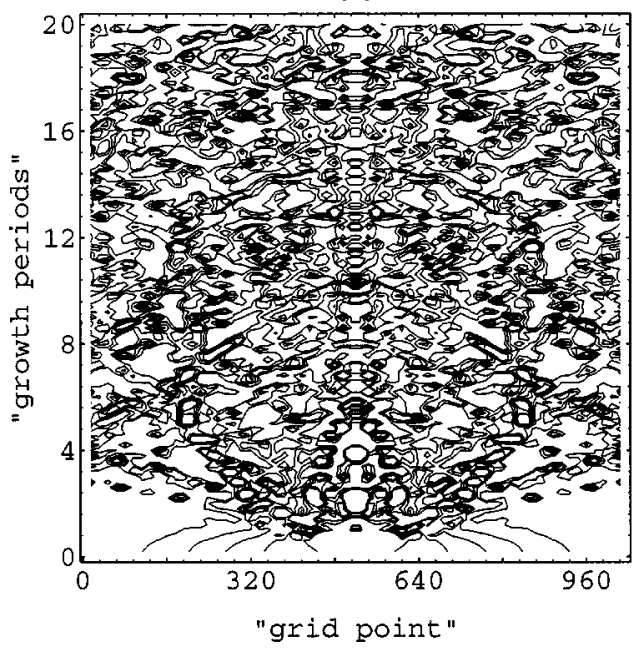

(e)

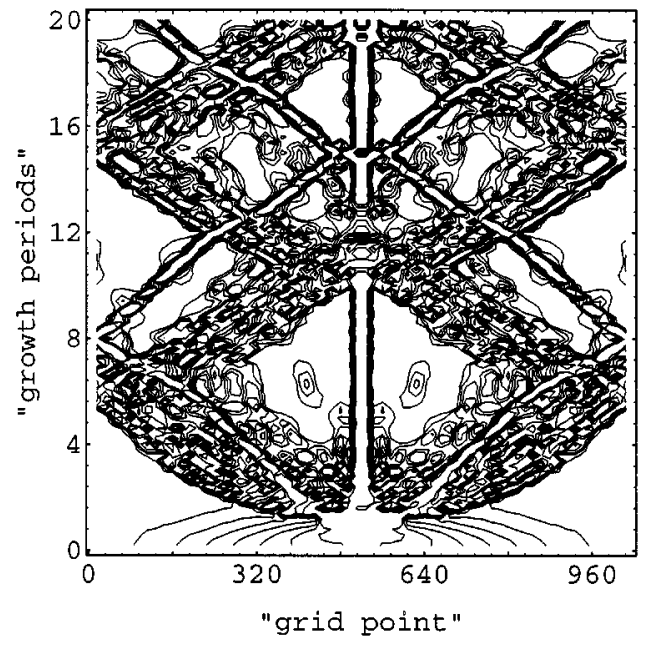

FIG. 4. Spatiotemporal simulations obtained with the full system. The figures display surface and contour plots of $|E(x, t)|^{2}$ and indicate how far solitons survive. (a) and (b) $k=k_{u} / 4$, (c) $k=k_{u} / 20$, (d) $k=k_{u} / 40$, and (e) the NLS spatiotemporal dynamics with $k=k_{u} / 20$ is displayed for the sake of comparison. $\rho_{*}=0.01$.

the value of $k$, the slower the growth. Accordingly, the similarity of the NLS and Zakharov systems is very much a matter of time, pump amplitude $\rho_{*}$, and basic wave vector $k$ for, since $\sqrt{\left\langle N^{2}\right\rangle_{Z}}$ is always growing, there will be a time when $\sqrt{\left\langle N^{2}\right\rangle_{Z}}$ and $\sqrt{\left\langle N^{2}\right\rangle_{Z}}$ become substantially different even if $k$ is not too small. Figure 5(c) really shows that the smaller $k$ is, the faster the diffusion. A question of relevance is to determine how the average growth of $\sqrt{\left\langle N^{2}\right\rangle_{Z}}$ scales 

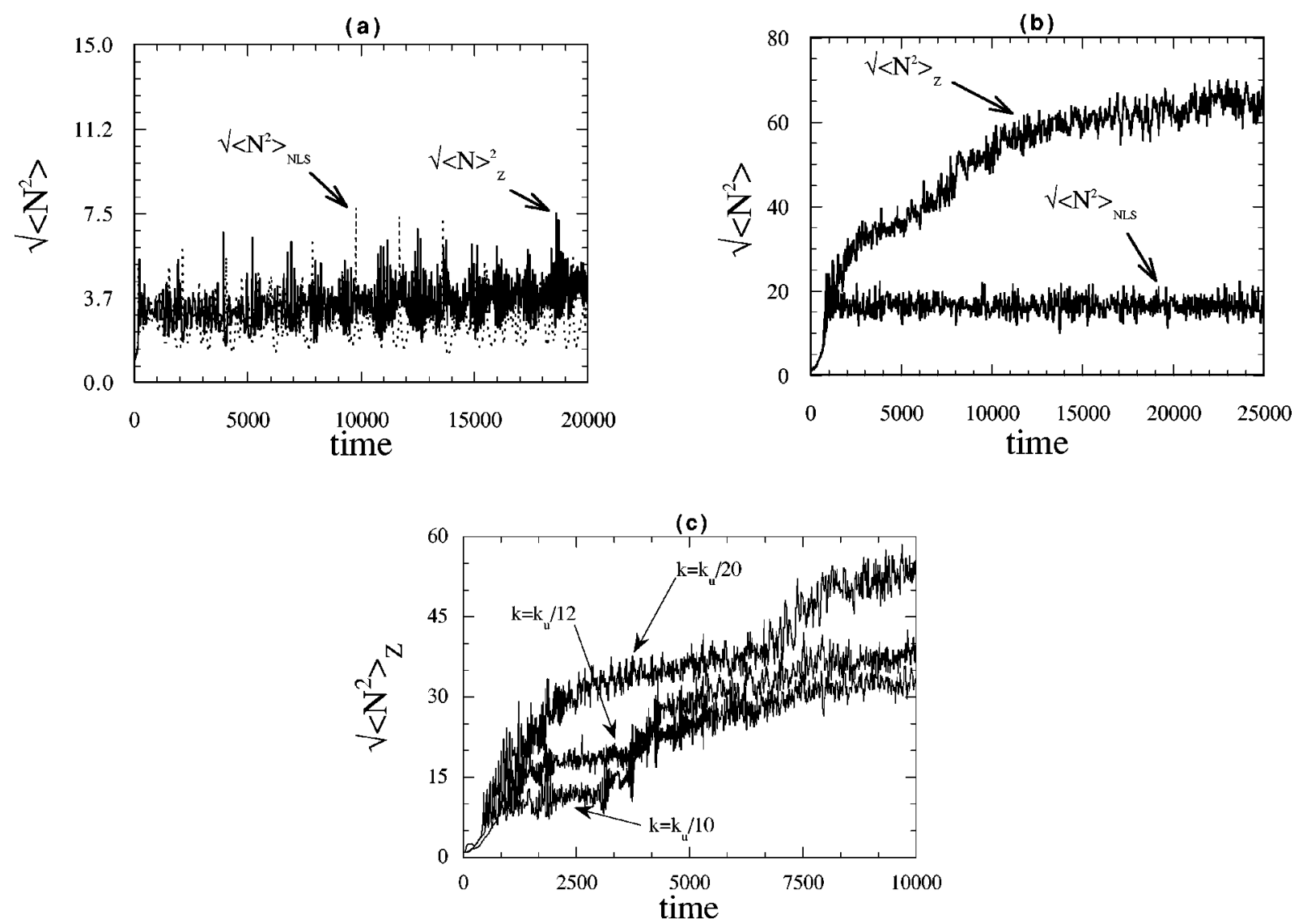

FIG. 5. Comparison of the spectral averages $\sqrt{\left\langle N^{2}\right\rangle_{Z}}$ and ${\sqrt{\left\langle N^{2}\right\rangle}}_{N L S}$ versus time for (a) $k=k_{u} / 4$ and (b) $k=k_{u} / 20$. In (c) we compare various curves representing $\sqrt{\left\langle N^{2}\right\rangle_{Z}}$ versus time. $\rho_{*}=0.01$.

with amplitude and wave vector. Issues like that require specific focus on computational statistics and are left for future works. In regard to this last point, we mention that to construct Fig. 5 (and Fig. 4 as well), our simulations attain convergence only with $N=1024$ (so we have to make runs with 2048 modes), which takes a couple of hours of CPU time on a Cray II. In any case, Fig. 5(c) shows that if one restricts attention to early times of the nonlinear interaction, for $\rho_{*}$ $=0.01$ no big discrepancy between the Zakharov and NLS equations occurs at higher values of $k$ than $k \approx k_{u} / 12$. This agrees with the results of previous simulations that used as the smallest value $k \sim k_{u} / 10$ [17]. In this particular case the $\sqrt{\left\langle N^{2}\right\rangle_{Z}}$ curve is quite similar to what would be obtained with the integrable NLS equation until $t \sim 5000$ when the number of modes really starts to pile up. To see the faster effects of the chaotic interaction one must really move to radically smaller values of $k$, which implies longer CPU times due to an increase in the number of modes needed in the simulations.

\section{FINAL REMARKS}

In this paper we have discussed energy transfer in the one-dimensional conservative Zakharov equations. The model is suitable to describe the time evolution of initial modulational instabilities in strongly magnetized systems. Radiation from pulsars also can be described by equations similar to the ones analyzed here.

Analysis was focused on small-amplitude fields, but even for small values of $\rho_{*}$, a transition to chaos is possible if $k$ is also chosen to be small enough (we have verified that for values of $\rho_{*}$ smaller than $\rho_{*}=0.01$ the transition is possible for appropriately smaller values of $k$ ). In this case soliton formation is eventually precluded and energy may freely flow into modes with smaller and smaller length scales. The way solitons respond to the ion-acoustic field is actually gradual, as seen here. In the typical conditions of our simulations where we considered wave vectors moderately small so as not to totally prohibit soliton formation, solitons are first formed as a result of the initial modulational instability. Only then, over longer time scales, are solitons affected by the free ion-acoustic radiation. They are affected according to the following rule: The smaller the value of $k$ the faster the energy flow seen in the full simulations and shorter the period of time where solitons can be really pinpointed on our space-time grids.

Under these conditions, the initial solitons can be seen as metastable structures exhibiting irregular fluctuations. As solitons undergo oscillatory motion they emit radiation. Here the oscillations are irregular and are maintained by the interaction with the ion-acoustic field. Solitons then emit over a broad spectral band, which causes a growing number of modes to be involved in the dynamics. This is in contrast to what happens in regular settings (the NLS equation, for instance) where solitons oscillate regularly until they settle down in a genuine solitary state [19]. As a matter of fact, solitons of nonintegrable system may never settle down sim- 
ply because the appropriate fixed point in the phase space could have been affected as a result of the associated chaotic dynamics. We point out that modes with small length scales appear in our system not because our solitary structures are collapsing. Quite on the contrary, because we have a onedimensional system and as can be seen from the effective solitonic potential of Eq. (20) with ion-acoustic terms discarded, collapse is absent. Therefore, small-scale modes can only appear when the dynamics goes deep into its nonintegrable regime and saturation via soliton formation is inhibited. In higher dimensions regular collapse leading to small scales is a possibility; in our case, however, soliton and soliton turbulence may not be very accurate models when $k$ is sufficiently small. These issues are under current investigation.

In recent years, a number of papers have shown that the final asymptotic state for the kind of interaction studied here can be, under certain conditions, a small number of solitons containing most of the energy of the system plus small fluctuations [20-23]. This small number of solitons would be in fact much smaller than the original number produced in the earlier stages of the interaction. What happens is that the various solitons generated initially may tend to keep fusing together until their number is drastically reduced. At this point the initial energy that was initially distributed over a relatively large number of structures becomes confined within this smaller number. It has been shown that the basic condition enabling fusion is that the soliton amplitudes are large enough [20]. Indeed, when $\rho \sim 1$ or larger, fusion takes place easily. In contrast, when $\rho \ll 1$ the time scale for fusion is presumably very long and the spatial scales smaller. We work with small energies that cause $\rho \ll 1$, which makes us believe that in terms of nonintegrable effects, the dominant feature here is the interaction of soliton oscillatory modes with ion-acoustic radiation. It is likely that after longer periods of time than those used in our simulations, smallamplitude solitons can also fuse together, although we have not yet explored this range of time. We emphasize that so far the effect of soliton breathing has not been considered in this context of nonintegrable features. As it appears, the beating of ion-acoustic and breathing oscillations leads to energy transfer in the case of small amplitudes.

\section{ACKNOWLEDGMENTS}

We are deeply thankful for stimulating discussions with L.P.L. de Oliveira. This work was partially supported by Financiadora de Estudos e Projetos and Conselho Nacional de Desenvolvimento Científico e Tecnológico, Brazil. Numerical computing was performed on the CRAY Y-MP2E at the Universidade Federal do Rio Grande do Sul Supercomputing Center.
[1] S.G. Thornhill and D. ter Haar, Phys. Rep. 43, 43 (1978).

[2] G.D. Doolen, D.F. DuBois, and H.A. Rose, Phys. Rev. Lett. 54, 804 (1985).

[3] H.T. Moon, Phys. Rev. Lett. 64, 412 (1990).

[4] X.T. He and C.Y. Zheng, Phys. Rev. Lett. 74, 78 (1995).

[5] S.E. Gibson, D.L. Newman, and M.V. Goldman, Phys. Rev. E 52, 558 (1995)

[6] G.I. de Oliveira, L.P.L. de Oliveira, and F.B. Rizzato, Phys. Rev. E 54, 3239 (1996).

[7] M. Pettini and M. Landolfi, Phys. Rev. A 41, 768 (1990).

[8] C.G. Goedde, A.J. Lichtenberg, and M.A. Lieberman, Physica D 59, 200 (1992).

[9] G. Tsaur and J. Wang, Phys. Rev. E 54, 4657 (1995).

[10] D.L. Shepelyansky (unpublished).

[11] A. Barnes and J.V. Holloweg, J. Geophys. Res. 79, 2302 (1974).

[12] A.C.-L. Chian and C.F. Kennel, Astrophys. Space Sci. 97, 9 (1983).

[13] D.U. Martin and H.C. Yuen, Phys. Fluids 23, 1269 (1980).

[14] A. Thyagaraja, Phys. Fluids 24, 1973 (1981).
[15] G.I. de Oliveira, L.P.L. de Oliveira, and F.B. Rizzato, Physica D 104, 119 (1997).

[16] M.M. Shen and D.R. Nicholson, Phys. Fluids 30, 1096 (1987).

[17] A.J. Lichtenberg and M.A. Lieberman, Regular and Stochastic Motion (Springer, New York, 1983).

[18] B. Malomed, D. Anderson, M. Lisak, M.L. Quiroga-Teixeiro, and L. Stenflo, Phys. Rev. E 55, 962 (1997).

[19] D. Anderson, M. Lisak, and T. Reichel, J. Opt. Soc. Am. B 5, 207 (1988).

[20] L.M. Degtyarev, V.G. Nakhan'kov, and L.I. Rudakov, Zh. Éksp. Teor. Fiz. 67, 533 (1974) [Sov. Phys. JETP 40, 264 (1974)].

[21] S.F. Krylov and V.V. Yan'kov, Zh. Éksp. Teor. Fiz. 79, 82 (1980). [Sov. Phys. JETP 52, 41 (1980)].

[22] A.I. D'yachenko, V.E. Zakharov, A.N. Pushkarev, V.F. Shvets, and V.V. Yan'kov, Zh. Éksp. Teor. Fiz. 96, 2026 (1989) [Sov. Phys. JETP 69, 1144 (1989)].

[23] M.B. Isichenko and A.V. Gruzinov, Phys. Plasmas 1, 1802 (1994). 